\title{
THE STRUCTURE AND DYNAMICS OF THE EARLY-TYPE RESONANCE RING GALAXY IC 4214. I. OBSERVATIONS

\author{
R. Buta, ${ }^{1,2}$ GuY B. Purcell, ${ }^{1,2}$ Melinda Lewis CobB, ${ }^{3}$ D. A. Crocker, ${ }^{1,2}$ \\ P. Rautiainen, ${ }^{4}$ and H. Salo ${ }^{4}$ \\ Received 1998 June 1; accepted 1998 October 12
}

\begin{abstract}
The southern galaxy IC 4214 is a bright, nearby example of a multiple ring early-type spiral galaxy with a weak bar. The galaxy shows three strong pseudoring features, including a nuclear ring, an inner ring, and a prominent $\mathrm{R}_{1}^{\prime}$ outer ring, each with distinctive characteristics. The galaxy is important because it is a classic resonance ring galaxy where the ring features can be linked to specific orbital resonances with the bar. We present in this paper a detailed $U B V I H$ study of its photometric structure and a Fabry-Perot study of its kinematics to set the stage for a dynamical model in a separate paper. Despite the early Hubble type, ionized gas is well distributed in the inner disk regions, providing a wellsampled velocity field. Rapid rotation is found in the vicinity of the nuclear ring, where the rotation curve reaches a maximum, and the rotation velocities drop off somewhat with increasing radius in the outer regions. Noncircular motions are clearly seen in the observed velocity field. Analysis of both the velocity field and the shapes of isophotes give discrepant values for the inclination of the system, ranging from $47^{\circ}$ to $50^{\circ}$ from photometry to $55^{\circ}$ to $58^{\circ}$ from kinematics. A likely value of the inclination could be $52^{\circ}$ if the inner and outer rings are intrinsically elongated and aligned perpendicular to each other.

Key words: galaxies: individual (IC 4214) — galaxies: kinematics and dynamics galaxies: photometry - galaxies: structure
\end{abstract}

\section{INTRODUCTION}

Barred galaxies are important topics of study for the following reasons: First, bars can be identified in $65 \%$ of normal, massive disk galaxies, based on blue light classifications (de Vaucouleurs 1963). This is likely to be a lower limit, since bars are usually made of old stars and are more prominent at longer wavelengths. In the near-infrared, the fraction of galaxies having bars is probably considerably higher than $65 \%$. Second, bars are well understood theoretically and provide an important and efficient engine for the secular evolution of structure in galaxies (Sellwood \& Wilkinson 1993; Martinet 1994). Spiral structure, rings, secondary bars, and "boxy bulges" can be connected in various ways to barred galaxy dynamics. Third, bars have been implicated in the development of nuclear activity in galaxies and in the weakening of abundance gradients, through the secular redistribution of gas (Simkin, Su, \& Schwarz 1980; Shlosman, Frank, \& Begelman 1989; Martin \& Roy 1994).

Rings are particularly important features of barred galaxies. The inner, outer, and nuclear rings seen in barred galaxies are the most identifiable tracers of orbital resonances in galaxies, and thus ringed galaxies have been the subject of many recent studies (see the review by Buta \& Combes 1996). What has been lacking in studies of ringed galaxies is dynamical analysis of individual cases having strong rings, clear bars, and optical and kinematic data.

\footnotetext{
${ }^{1}$ Department of Physics and Astronomy, University of Alabama, Tuscaloosa, AL 35487-0324.

${ }^{2}$ Visiting Astronomer, Cerro Tololo Inter-American Observatory, National Optical Astronomy Observatories, which is operated by the Association of Universities for Research in Astronomy (AURA), Inc., under cooperative agreement with the National Science Foundation.

${ }^{3}$ Department of Physics and Astronomy, Muskingum College, New Concord, OH 43762.

${ }^{4}$ Department of Physical Sciences, Division of Astronomy, University of Oulu, FIN-90570 Oulu, Finland.
}

Such studies are needed to evaluate the resonance identifications that have been provided by comparisons between morphology and test particle models (Buta 1995) and to provide dynamical information such as pattern speeds, dark halo contributions, and relative bulge to disk masses.

In this paper, we present a detailed study of IC 4214, one of the nearest and brightest examples of a multiple resonance ring galaxy in the southern sky. IC 4214 is a threering system with very regular structure and only a weak bar. The galaxy provides a good comparison between barred galaxy theory and observations because its rings are well defined and there is diffuse ionized gas in the inner regions that gives the crucial information on global kinematics needed for any serious model. The galaxy has no massive nearby companions of similar redshift, so that its structure can be studied from the point of view of internal dynamics alone. The galaxy has also been previously studied in detail by Saraiva (1997), who noted the galaxy's many similarities to other early-type ringed galaxies. The present paper covers only the observational details of our study, some of which overlaps the work of Saraiva. In the following paper (Salo et al. 1999, hereafter Paper II), we present our dynamical model of the galaxy based on a two-dimensional sticky particle code. Similar observational studies of five other resonance ring galaxies that can be compared with IC 4214 are provided by Buta \& Purcell (1998) and Buta et al. (1998).

\section{OBSERVATIONS AND REDUCTIONS}

For this project we used three sets of observational data: optical $U B V I$ CCD images, near-infrared $H$-band images, and imaging Fabry-Perot interferometry, all obtained at Cerro Tololo Inter-American Observatory with the 1.5 and $4 \mathrm{~m}$ telescopes. Several observational parameters and details are listed in Table 1. The instrumental setup for the optical images is described by Buta \& Crocker (1991). A total of 10 optical images was obtained, one in $U$ and three each in $B, V$, and $I$. The final $B, V$, and $I$ images are mosaics 
TABLE 1

OBSERVATIONAL PARAMETERS

\begin{tabular}{|c|c|c|c|c|c|c|c|c|c|}
\hline Instrument & $\begin{array}{l}\text { Telescope } \\
\text { (m) }\end{array}$ & Date & Filter & $\begin{array}{l}\lambda_{\text {eff }} \\
(\mu \mathrm{m})\end{array}$ & $\begin{array}{c}\text { Scale } \\
\left(\operatorname{arcsec} \text { pixel }^{-1}\right)\end{array}$ & $\begin{array}{c}\text { Gain } \\
\left(e^{-} \mathrm{ADU}^{-1}\right)\end{array}$ & $\begin{array}{l}\mathrm{RN}^{\mathrm{a}} \\
\left(e^{-}\right)\end{array}$ & $\begin{array}{l}\text { Exposure } \\
\text { (s) }\end{array}$ & $\begin{array}{c}\text { FWHM }^{\mathrm{b}} \\
\left({ }^{\prime \prime}\right)\end{array}$ \\
\hline TI No. 3 CCD....... & 1.5 & $1990 \mathrm{Feb}$ & $U$ & 0.35 & 0.547 & 1.7 & 5.9 & 480 & 1.4 \\
\hline TI No. 3 CCD...... & 1.5 & $1990 \mathrm{Feb}$ & $B$ & 0.44 & 0.547 & 1.7 & 5.9 & 780 & 1.7 \\
\hline TI No. 3 CCD...... & 1.5 & $1990 \mathrm{Feb}$ & $V$ & 0.55 & 0.547 & 1.7 & 5.9 & 450 & 1.5 \\
\hline TI No. 3 CCD...... & 1.5 & $1990 \mathrm{Feb}$ & $I$ & 0.8 & 0.547 & 1.7 & 5.9 & 270 & 1.7 \\
\hline CIRIM $^{\mathrm{c}} \ldots \ldots \ldots \ldots$ & 1.5 & $1996 \mathrm{Feb}$ & $H$ & 1.6 & 1.142 & 9 & 37 & 1200 & 2.0 \\
\hline $\operatorname{RFP}^{d} \ldots \ldots \ldots \ldots \ldots$ & 4.0 & $1992 \mathrm{Mar}$ & $\mathrm{H} \alpha$ & 0.66 & 0.394 & 2.5 & 7 & $600 /$ frame & 3.0 \\
\hline
\end{tabular}

${ }^{\text {a }}$ Read noise.

${ }^{\mathrm{b}}$ Full width at half-maximum.

${ }^{c}$ CTIO Infrared Imager.

d Rutgers Fabry-Perot Inteferometer.

of three images obtained in each filter to provide accurate sky subtraction. All flat-fielding, bias subtraction, cosmicray removal and image combinations were performed using IRAF software. ${ }^{5}$

Calibration of the optical images was performed using both faint E-region standards (Graham 1982) and equatorial standards (Landolt 1992). Buta \& Crocker (1992) published photoelectric UBVRI photometry of IC 4214 within four different circular apertures. As a check on our calibrations, we have integrated the fluxes from our arrays within the same apertures and compared the fully transformed values with the photoelectric values. Only the three smaller apertures are reliable for the comparison, owing to the bright star close to and southeast of the center that affected the largest aperture. From the smaller apertures, the mean differences found are $\left\langle U-U_{\mathrm{pe}}\right\rangle=+0.084 \pm$ $0.016,\left\langle B-B_{\mathrm{pe}}\right\rangle=-0.027 \pm 0.019,\left\langle V-V_{\mathrm{pe}}\right\rangle=-0.024$ $\pm 0.010,\left\langle I-I_{\mathrm{pe}}\right\rangle=-0.007 \pm 0.004$, where pe refers to the photoelectric values. Except for the $U$ band, this is quite satisfactory agreement.

The instrumental setups and reduction procedures for the Fabry-Perot and $H$-band images are the same as described by Buta \& Purcell (1998) and will not be repeated here. Owing to the high inclination of IC 4214, it was necessary to obtain 19 Fabry-Perot frames in order to cover the whole velocity range in the galaxy. A few of these frames were affected by night sky emission, probably due to $\mathrm{O}$ I $\lambda 6603$. The rings from this emission were removed using a sky fitting algorithm provided by T. B. Williams. Calibration of the Fabry-Perot frames was performed by computing radii and centers for a series of rings based on imaging of several calibration lamp lines at different etalon spacings. Calibration of the $H$-band data was performed by observing three standard stars from Elias et al. (1982). There is no published $H$-band aperture photometry of IC 4214 that could be used to check our zero point, but other galaxies observed during the same run that do have such photometry available (see Buta \& Purcell 1998 and Buta et al. 1998) indicate possible systematic disagreements of $0.1-0.2$ mag.

The scale and orientation of all images used for this study were determined astrometrically using stars from the Space Telescope Science Institute (STScI) Guide Star Catalog as

\footnotetext{
${ }^{5}$ IRAF is distributed by National Optical Astronomy Observatories, which are operated by the Association of Universities for Research in Astronomy, under cooperative agreement with the National Science Foundation.
}

positional standards. Since few such stars were available on the actual frames, we established our own positional standards on them by bootstrapping Guide Star Catalog positions from a wider field frame obtained from the Digitized Sky Survey. The scales derived are listed in Table 1. Based on this astrometry, all position angles in this paper are converted to a B1950.0 coordinate system.

\section{MORPHOLOGY AND REDSHIFT DISTANCE}

Table 2 summarizes available type estimates for IC 4214 from the Catalog of Southern Ringed Galaxies (CSRG; Buta 1995), the Southern Galaxy Catalog (SGC; Corwin, de Vaucouleurs, \& de Vaucouleurs 1985), the ESO B catalogue (Lauberts 1982), and Buta \& Crocker (1991, hereafter BC91). There is general agreement on the classification as an early-type spiral galaxy around type Sa. Although Corwin et al. (1985) and Lauberts (1982) classify the family as type SB, Buta (1995) and BC91 did not distinguish a clear bar in blue light. However, a weak bar is definitely seen in $I$-band images (see Fig. 7 of Saraiva 1997).

Figure 1 illustrates $B$ - and $H$-band images of IC 4214 that have been matched in scale and orientation using the IRAF routines GEOMAP and GEOTRAN. As also noted by BC91, the $B$-band image shows that IC 4214 is a classic double-ringed galaxy with strong inner and outer rings. Both rings actually appear to be pseudorings made of tight spiral structure. The outer ring has a morphology consistent with a theoretical Schwarz (1981) model A outer pseudoring, that is, a type where the outer arms wind about $180^{\circ}$ with respect to the ends of the bar (see Fig. 4 of Schwarz 1981). BC91 classify such a pseudoring as type $R_{1}^{\prime}$. The $B$-band image also shows the nuclear spiral or pseudoring initially recognized by BC91. In the $H$ band the structure is much smoother, consistent with an emphasis on an older stellar population, but all three ring features can still be distinguished. There is no clear evidence for a secondary bar inside the nuclear ring, even in the $H$ band (see $\S 6$ ).

The large-scale $B-I$ color distribution in IC 4214 was illustrated by BC91. In Figure 2, we show a montage of color index maps of the region covering only the inner pseudoring and central section (see also Saraiva 1997). All maps are matched to the resolution and seeing of the $H$-band image (see Table 1 for the FWHM of the different images). The $U-B$ and $B-V$ maps illustrate well the distribution of star formation in the tightly wrapped spiral arms of the inner and nuclear pseudorings. The dust distribution is best illustrated in $B-I$ and especially $I-H$. The latter color, in particular, shows a circumnuclear dust ring and two curved 
TABLE 2

Global Optical Characteristics of IC 4214

\begin{tabular}{|c|c|}
\hline Parameter & Value \\
\hline$\alpha(\mathrm{B} 1950.0)^{\mathrm{a}}$ & 1314561 \\
\hline$\delta(\mathrm{B} 1950.0)^{\mathrm{a}}$ & -315018 \\
\hline SGC type $^{\mathrm{b}} .$. & $\left(\mathrm{R}^{\prime}\right) \mathrm{SB}(\mathrm{r}) \mathrm{ab}$ \\
\hline CSRG type ${ }^{c}$. & $\left(\mathrm{R}_{1}^{\prime}\right) \mathrm{S} \underline{\mathrm{AB}}(\mathrm{r}) \mathrm{a}$ \\
\hline ESO-B type ${ }^{\mathrm{d}}$ & $\mathrm{SB}(\mathrm{r}) 0(\mathrm{r})$ \\
\hline Other type ${ }^{\mathrm{e}} \ldots \ldots \ldots$ & $\left(\mathrm{R}_{1}^{\prime}\right) \mathrm{SA}(\mathrm{r}) \mathrm{a}$ \\
\hline 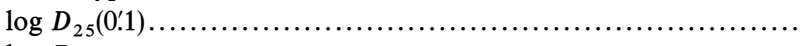 & $1.466 \pm 0.013$ \\
\hline $\log R_{25} \ldots \ldots \ldots \ldots \ldots \ldots$ & $0.162 \pm 0.016$ \\
\hline$\phi_{25}(\mathrm{~B} 1950.0)(\mathrm{deg}) \ldots \ldots$. & $173 \pm 3$ \\
\hline $\log A_{e}(0,1) \ldots \ldots \ldots \ldots \ldots$ & $0.89 \pm 0.02$ \\
\hline$B_{T} \ldots \ldots \ldots \ldots \ldots \ldots \ldots \ldots \ldots$ & $12.22 \pm 0.03$ \\
\hline$(B-V)_{T} \ldots \ldots \ldots \ldots \ldots \ldots$ & $0.83 \pm 0.02$ \\
\hline$(U-B)_{T} \ldots \ldots \ldots \ldots \ldots$ & $0.41 \pm 0.02$ \\
\hline$(V-I)_{T} \ldots \ldots \ldots \ldots \ldots \ldots \ldots$ & $1.15 \pm 0.03$ \\
\hline$(B-H)_{T} \ldots \ldots \ldots \ldots \ldots \ldots$ & $3.96 \pm 0.04$ \\
\hline$(B-V)_{e} \ldots \ldots$ & $0.90 \pm 0.01$ \\
\hline 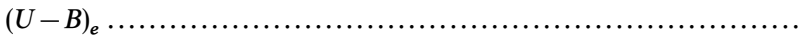 & $0.43 \pm 0.01$ \\
\hline (................................. & $1.23 \pm 0.01$ \\
\hline 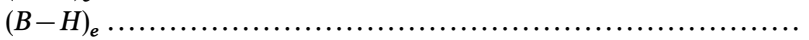 & $4.17 \pm 0.02$ \\
\hline Photometric axis ratio $\left\langle q_{p}\right\rangle\left(70^{\prime \prime} \leq r \leq 90^{\prime \prime}\right) \ldots \ldots \ldots \ldots \ldots \ldots \ldots \ldots \ldots$ & $0.698 \pm 0.009$ \\
\hline Photometric major axis $\left\langle\phi_{p}\right\rangle\left(70^{\prime \prime} \leq r \leq 90^{\prime \prime}\right)(\mathrm{B} 1950.0)(\mathrm{deg}) \ldots \ldots$ & $175.0 \pm 1.4$ \\
\hline Photometric inclination $i_{p}\left(q_{0}=0.2 \pm 0.1\right)(\mathrm{deg}) \ldots \ldots \ldots \ldots \ldots \ldots \ldots \ldots$ & $47 \pm 2$ \\
\hline Heliocentric systemic radial velocity $v_{s, \odot}\left(\mathrm{km} \mathrm{s}^{-1}\right) \ldots \ldots \ldots \ldots \ldots$ & $2310 \pm 2$ \\
\hline 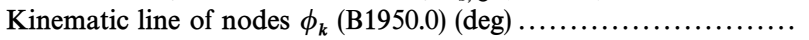 & $169 \pm 1$ \\
\hline 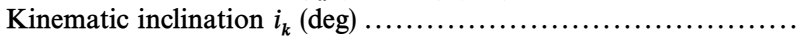 & $57 \pm 2$ \\
\hline
\end{tabular}

NoTE.- Units of right ascension are hours, minutes, and seconds, and units of declination are degrees, arcminutes, and arcseconds.

${ }^{\text {a }}$ De Vaucouleurs et al. 1991.

${ }^{\text {b }}$ Corwin et al. 1985.

c Buta 1995.

d Lauberts 1982.

e Buta \& Crocker 1991.

dust lanes reminiscent of what is seen in barred spiral galaxies of type $\mathrm{SBb}$. The northwest dust lane is most prominent in optical colors, suggesting that the west side is the near side. Referring to our velocity field (§ 5.2), this implies that the spiral arms are trailing, which places the dust lanes seen in $I-H$ on the leading edges of the weak bar. The strong curvature of the dust lanes is qualitatively consistent with expectations for a weak bar (Athanassoula 1992).

The deep images also reveal a possible dwarf companion superposed nearly due east of the center of IC 4214 (see Fig. 3 ). This feature represents a distortion of the outer isophotes, but it is not clear that it is merely a distortion of IC 4214. If it is a dwarf, it would be reminiscent of the unusual companion of NGC 7531 (Buta 1987). The position of the center of the feature is approximately $62^{\prime \prime}$ east and $5^{\prime \prime}$ north of the nucleus of IC 4214 , corresponding to $\alpha=13^{\mathrm{h}} 15^{\mathrm{m}} 01^{\mathrm{s}}$, $\delta=-31^{\circ} 50^{\prime} 13^{\prime \prime}(\mathrm{B} 1950.0)$.

The heliocentric radial velocity of IC 4214 is $v_{\odot}=2310$ $\mathrm{km} \mathrm{s}^{-1}$, based on the Fabry-Perot interferometry described in $\S 5$. This agrees well with the value $2281 \pm 23 \mathrm{~km} \mathrm{~s}^{-1}$ given in de Vaucouleurs et al. (1991). Using the linear Virgocentric flow model of Aaronson et al. (1982), we estimate a distance 1.86 times that of the Virgo Cluster, which would correspond to $31.6 \pm 3.7 \mathrm{Mpc}$ if the distance to Virgo is $17 \pm 2 \mathrm{Mpc}$ (see discussion in Buta et al. 1998). The broadband angular diameters of the three ring features are given by Buta \& Crocker (1993). For a distance of $31.6 \mathrm{Mpc}$, the linear diameters would be $18,8.4$, and $1.3 \mathrm{kpc}$ for the outer, inner, and nuclear rings, respectively, which are typical values.

\section{SURFACE PHOTOMETRY}

\subsection{Foreground Star Removal and Isophote Fitting}

Before using the images for surface photometry, it was necessary to clean the images of foreground stars. For most of these stars, a routine similar to IMEDIT in IRAF was used. A star would be assigned the diameter of a circular patch of specific radius, and a fit would be made to intensity values within a circular annulus of specified width. The fit would then be interpolated over the area of the star, and the interpolations would replace the star image. Although this approach generally works extremely well, it could not be used for the bright star superposed on the southeast end of the galaxy. This star saturated on the $B, V$, and $I$ images and developed a significant off-center halo that could not be satisfactorily interpolated over. To deal with this star, we used two approaches. One was to mask out the large area affected by the star and its off-center halo by replacing the pixels with a large intensity value that an ellipse-fitting code would ignore. Since the star is close to the major axis, a large chunk of this axis on one side is effectively eliminated from any ellipse fits to isophotes. In the second method, the bright star was treated by replacing the affected region with intensities from symmetrically located points on the opposite side of the galaxy. This seemed a reasonable 

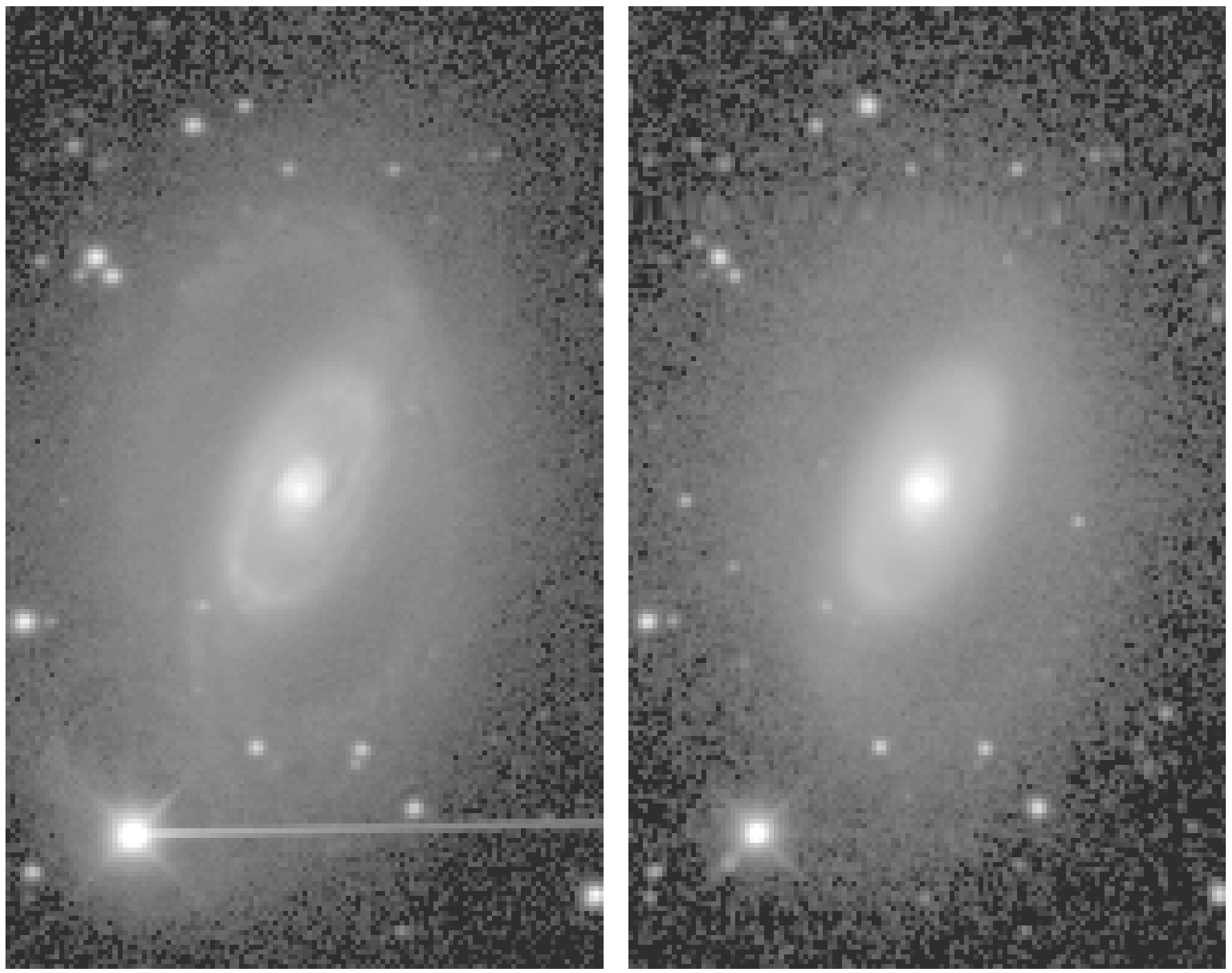

FIG. 1.-Images of IC 4214 in $B$ (left) and $H$ (right). The units of the images are mag arcsec ${ }^{-2}$ ranging from 17 to $28 \mathrm{mag}^{-\operatorname{arcsec}^{-2}}$ in $B$ and from 13.5 to $24.0 \mathrm{mag} \operatorname{arcsec}^{-2}$ in $H$. The field shown in each frame covers $3^{\prime} .8 \times 2 \cdot 3$. North is at the top, and east is to the left.

approach since the system is not interacting and not very highly inclined.

Ellipse fits to the isophotes were made using two routines: a general least-squares routine, which solves for the center-, major-, and minor-axis radii and major-axis position angle for an isophote at specified surface brightness; and the routine ELLIPSE in the IRAF reduction package, which uses Fourier techniques to find the best-fitting ellipse corresponding to a specified radius (Jedrzejewski 1987). The latter was modified to work for nonmonotonically declining surface brightness distributions by W. Freudling, who made the modification available to the astronomical community as the routine SPHOT in the IRAF-based GALPHOT package. SPHOT is equipped to handle the large masked region over the bright star, and Fourier techniques are probably best suited to minimize the effects of the missing portions of isophotes due to this star. The other ellipsefitting routine, called SPRITE, is a local IRAF-compatible program and was used to fit the image where the star was replaced by reflection of points from the opposite side of the galaxy.

The results for both approaches as applied to $B, V, I$, and $H$ filters are illustrated in Figure 4. The orientation of a system like IC 4214 is never easy to determine since axisymmetry cannot necessarily be assumed for any part of the visible disk. Nevertheless, since the isophotal axis ratio is relatively constant in the radius range $70^{\prime \prime} \leq a \leq 90^{\prime \prime}$, we use this range to estimate a photometric axis ratio and major-axis position angle. From the SPRITE fits to the reflected images, the mean axis ratio and position angle over this range are 0.698 and $175^{\circ}$, respectively. From the SPHOT fits to the masked images, the mean axis ratio and position angle over the same range are 0.687 and $172^{\circ}$, respectively. Thus, the two approaches are in fairly good agreement. The reflection images provide the best consistency between the different filters, and we tentatively adopt the results from the SPRITE fits. These are listed as the photometric position angle $\phi_{p}$ and axis ratio $q_{p}$ in Table 2 . Assuming the bulge is insignificant in this region, the intrinsic disk axis ratio is $q_{0}=0.2$ (Aaronson, Huchra, \& Mould 1980 ), and also that the isophotes in the above radius range define an oblate axisymmetric zone, then the inclination is $47^{\circ}$ based on Hubble's formula (Hubble 1926). According to Schommer et al. (1993), the additive correction of $+3^{\circ}$ advocated by Aaronson et al. (1980) may not be appropriate for an axis ratio based on ellipse fits to CCD data over a 

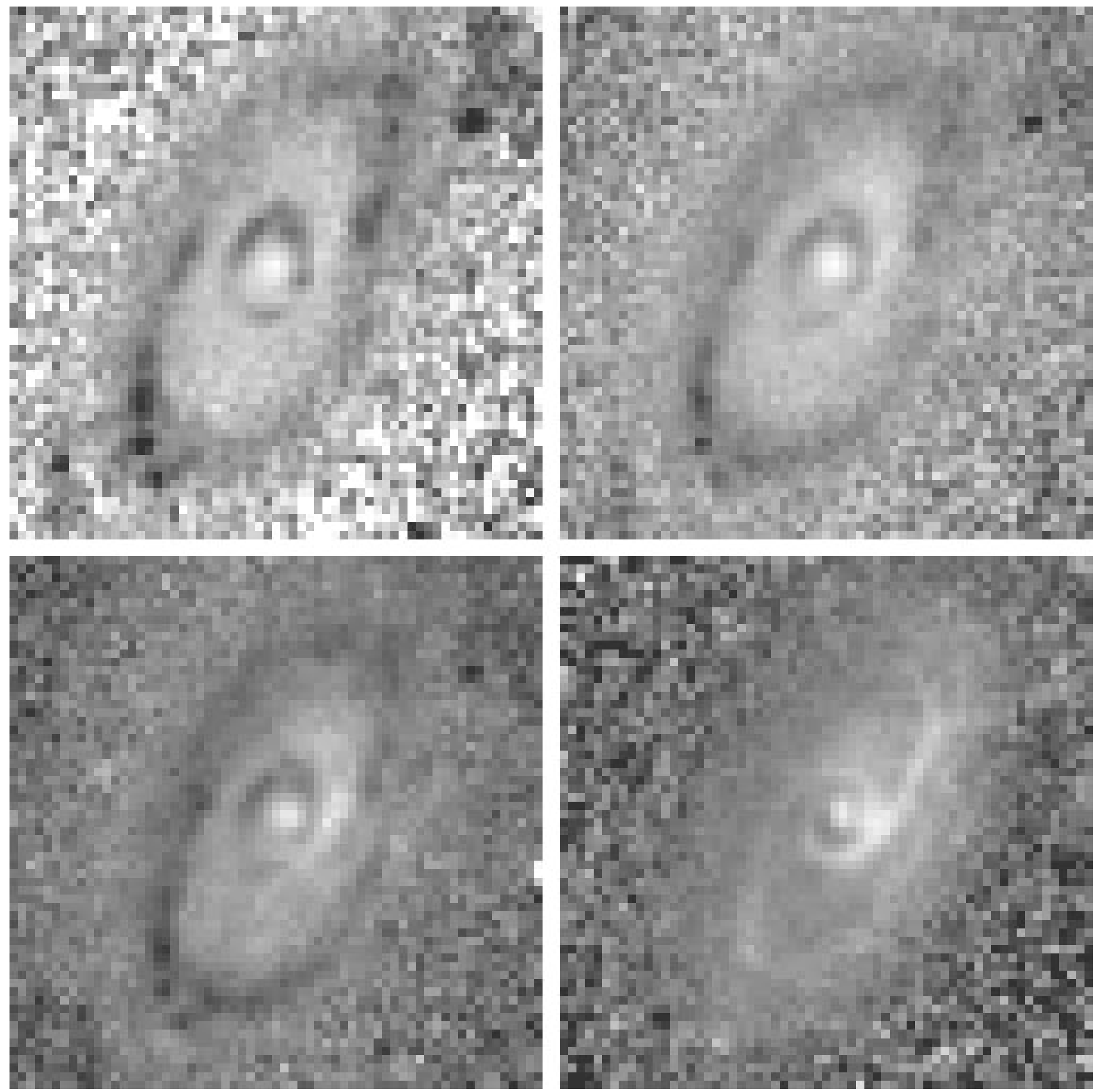

Fig. 2.-Montage of color index maps of IC 4214. The panels show $U-B$ (top left); $B-V$ (top right); $B-I$ (bottom left); and $I-H$ (bottom right). The gray scales are such that redder features are light and bluer features are dark. The ranges displayed are $-0.25-0.80$ for $U-B, 0.5-1.2$ for $B-V, 1.5-2.85$ for $B-I$, and 1.5-2.5 for $I-H$. Each frame covers 1'.22 $\times 11^{\prime} 22$. North is at the top and east is to the left.

range of surface brightnesses, and therefore we have not applied a correction. If the uncertainty in $q_{0}$ is \pm 0.1 , then the uncertainty in $i$ from this formula would be only 1.5 . This would be a minimum uncertainty, since the assumption of oblateness and axisymmetry in the zone where $q_{p}$ was measured may be incorrect.

\subsection{Basic Photometric Parameters and Surface Brightness Profiles}

Table 2 lists basic parameters derived from the broadband images of IC 4214, including the total magnitude $B_{T}$, the effective aperture $A_{e}$ enclosing one-half the total $B$-band flux, the integrated and effective $B-I$ colors, and the isophotal parameters at the standard surface brightness level of $\mu_{B}=25.00 \mathrm{mag} \operatorname{arcsec}^{-2}$. The standard isophotal radius, $D_{25} / 2=88^{\prime \prime}$, is within the range of radius used to estimate $q_{p}$ and $\phi_{p}$ above. If we use the axis ratio of this isophote alone and equation (2) of Fouqué et al. (1990) to derive $q_{0}$, then the inclination would be $50^{\circ}$. Fouque et al. note that their relation for the morphological type dependence of $q_{0}$ differs from previous relations (e.g., Bottinelli et al. 1983) and predicts higher inclinations than those relations. 


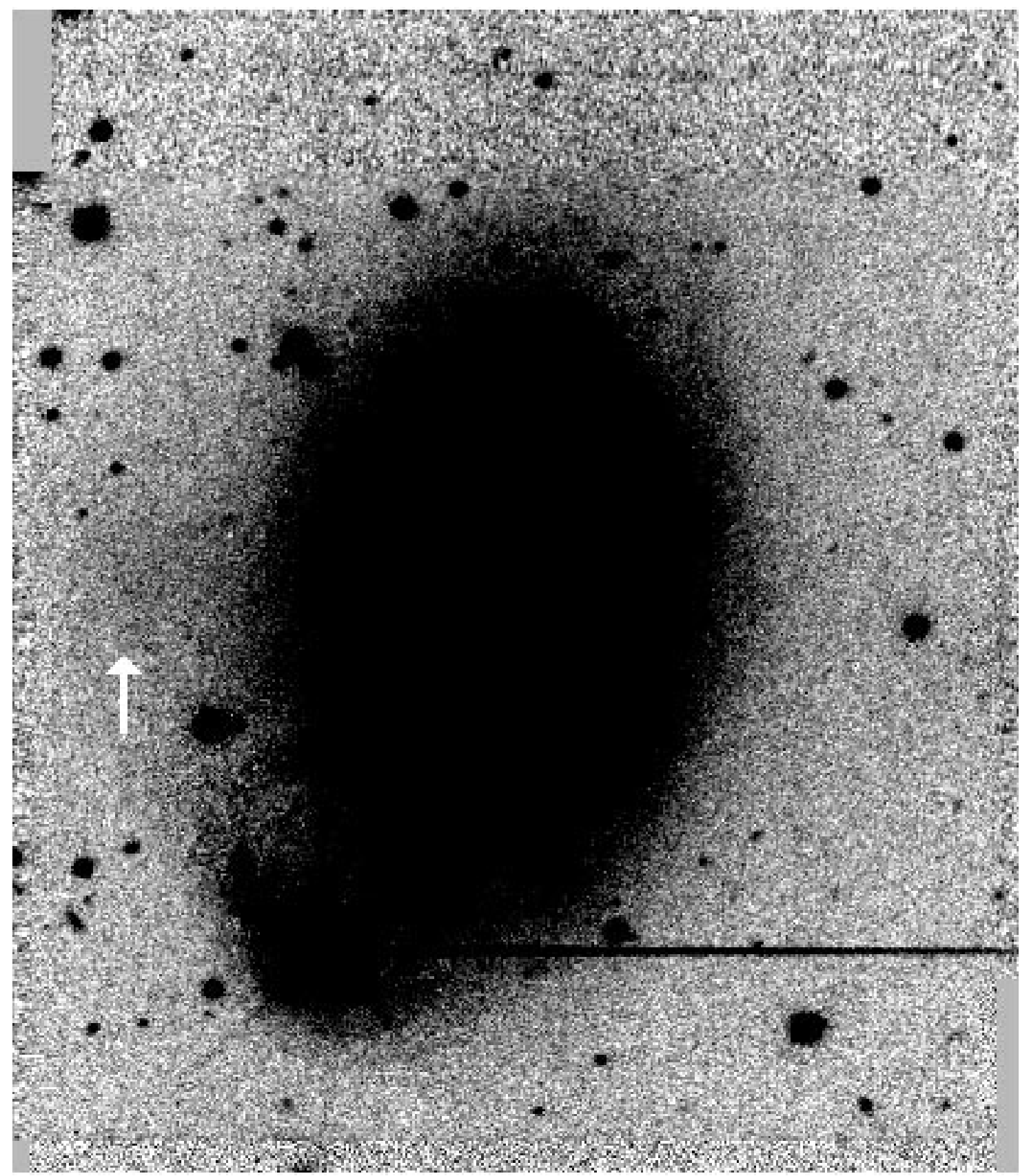

FIG. 3.-Deep $B$-band negative image of IC 4214 showing the faint possible dwarf companion (arrow) superposed to the east. The field shown covers $4: 3 \times 3: 7$. North is at the top, and east is to the left.

Figure 5 shows luminosity and color profiles along position angles $175^{\circ}$ and $85^{\circ}$, the photometric major and minor axes of the disk. Optical profiles are in Figures $5 a$ and $5 b$, while $H$-band profiles are in Figures $5 c$ and $5 d$. The profiles are typical of an early-type ringed galaxy, and their considerable symmetry in $H$ compared with $B$ is noteworthy. Dust and recent star formation seriously affect the optical light profiles. The major-axis structure to larger radii is illustrated with the folded profiles ( filled circles) in Figure 6. Each ring produces a prominent "bump" in the folded $B$-band profile. The surface brightness of the outer ring is $\mu_{B} \approx 23.5 \mathrm{mag} \operatorname{arcsec}^{-2}$, making it one of the brightest known examples. The surface brightnesses of the inner and nuclear rings are typical (Buta \& Combes 1996).

The deepest profiles are shown by the solid curves in Figure 6 . These are elliptically averaged profiles, where the averages were taken within an ellipse defined by $q_{p}$ and $\phi_{p}$ in Table 2 . These indicate that the average colors are nearly uniform beyond $r=60^{\prime \prime}$, the approximate radius of the outer ring.

\subsection{Bulge Model}

In the analysis of the gravitational potential in Paper II, we need to allow for the smaller flattening of the bulge in IC 4214 compared with the disk. If all of the material is assumed to be confined to a thin disk, deprojection will stretch the bulge isophotes into an artificial bar, complicating analysis of the gas flow. We have attempted to decompose the bulge from the disk component using the iterative decomposition method of Kent (1986). Although conventional fitting functions (exponential disk, $r^{1 / 4}$ bulge) can provide a reasonable-looking model of the profile along the 

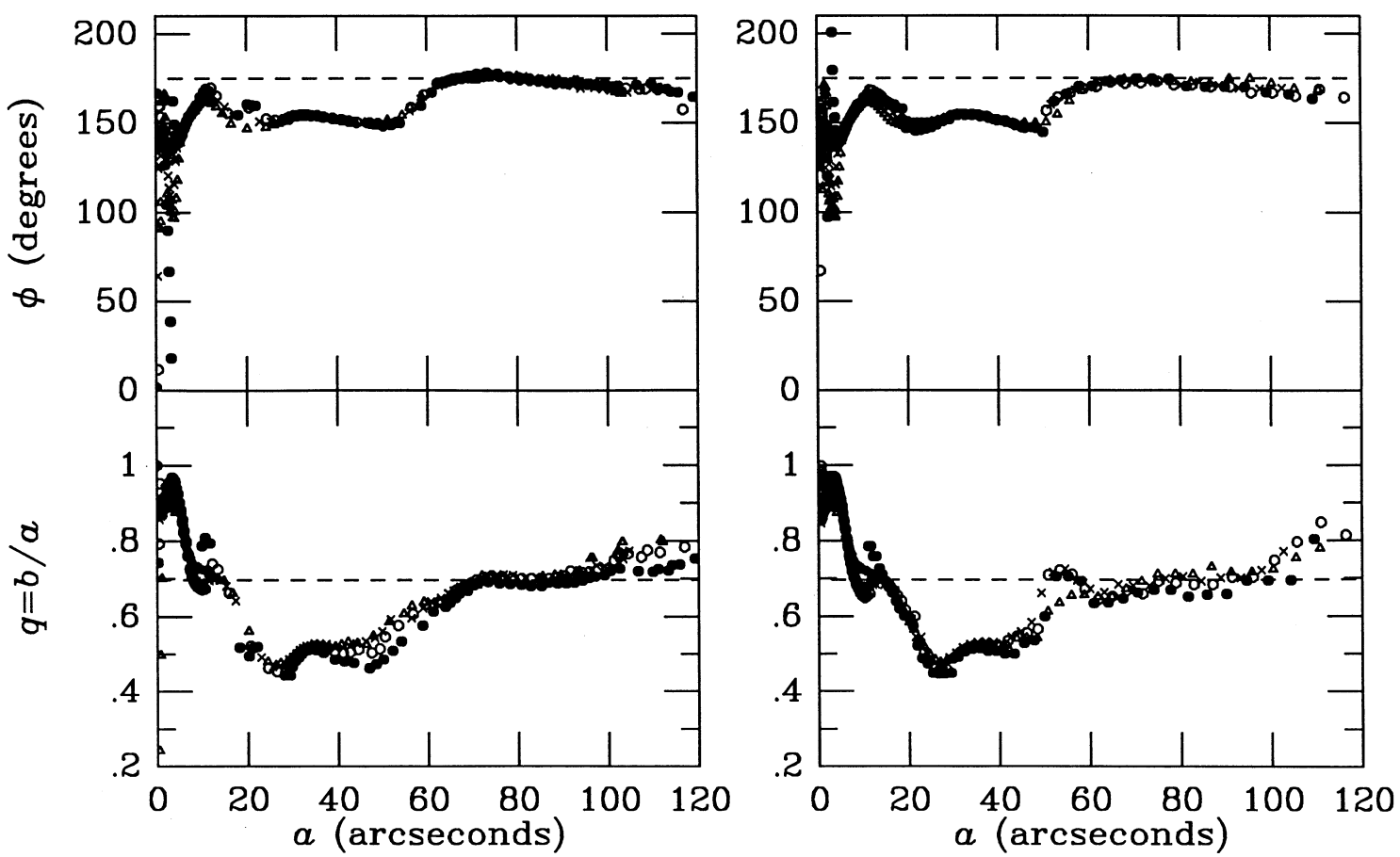

FIG. 4.- Results of ellipse fits to isophotes of IC 4214. The plots show the axis ratio $q$ and position angle $\phi$ vs. major-axis radius $a$. Filled circles are $B$, open circles are $V$, crosses are $I$, and open triangles are $H$. The left panel shows the results of SPRITE ellipse fits to the images where the area affected by the bright star to the southeast was replaced with intensities from the opposite side of the galaxy. The right panel shows the results of SPHOT ellipse fits to the images after the bright star was masked instead. The dashed horizontal lines are the adopted values from Table 1.

major axis (see Fig. 13 of Saraiva 1997), such models do not project well to the minor axis. Kent's method involves a comparison of major- and minor-axis profiles that takes advantage of the different projected shapes of the bulge and disk, with the bulge usually being less flattened. However, the intrinsically oval rings in IC 4214 complicate application of the method.

We show the results of Kent's method as applied to our $H$-band profiles as the solid curves without points in Figures $5 c$ and $5 d$. For the analysis we used a disk flattening of 1/0.698 and a bulge flattening of 1.0. For these flattenings, the method provides a reasonably smooth bulge profile for $r<10^{\prime \prime}$. At larger radii, the derived profile is not reliable because of the complex nonaxisymmetric structure and is not shown. For $4^{\prime \prime} \leq r \leq 10^{\prime \prime}$, the bulge profile is approximately exponential, while for $r<4^{\prime \prime}$, the slope is less steep. A somewhat better fit to the observed surface brightnesses for $r<2^{\prime \prime}$ can be obtained if we set the bulge flattening to slightly less than 1 (making the bulge elongated perpendicular to the major axis). To improve the bulge model at smaller radii, a higher resolution $H$-band image is needed.

\section{IMAGING FABRY-PEROT INTERFEROMETRY}

\subsection{H II Region Distribution}

Imaging Fabry-Perot interferometry provides three maps: net line intensity, velocity field, and velocity dispersion. The line intensity map of IC 4214 is shown next to a global $U-I$ color index map in Figure 7. There are several interesting features of the $\mathrm{H} \alpha$ distribution. First, the nuclear and inner pseudorings are more ringlike in $\mathrm{H} \alpha$ than they are in blue light. The nuclear ring is very bright and has a slightly oval ridge line. The inner ring is a very oval feature and is lined by bright $\mathrm{H}$ II regions along the prominent spiral arcs in blue light. There is diffuse emission underlying the inner ring region. The outer ring is lined by bright $\mathrm{H}$ II regions along trailing spiral arcs from the major-axis region of the inner ring. Most interesting is the location of two prominent $\mathrm{H}$ II regions near dimple points in the outer ring (see arrows in Fig. 7, right). These are also seen in the $U-I$ map; they lie off the ends of the inner ring and may be connected to a slowing down of gas in these regions (see Fig. 4 of Schwarz 1981). The apparently brightest H II region in IC 4214 is, in fact, located at the western dimple point.

Dimple $\mathrm{H}$ II regions are not commonly observed in outerringed galaxies. Crocker, Baugus, \& Buta (1996) found few examples in a sample of 32 ringed galaxies, several of which possess strong $\mathrm{R}_{1}^{\prime}$ outer pseudorings.

\subsection{Kinematics and Rotation Curve}

Figure 8 shows the $\mathrm{H} \alpha$ velocity field of IC 4214 . The map includes only those points for which the fitted velocity has a formal error of $\leq 10 \mathrm{~km} \mathrm{~s}^{-1}$. The velocity field is well sampled in the inner ring region owing to the diffuse emission, and the contours show a definite twisting in that region. In Paper II, our model shows that this is attributable to oval streamlines in the bar region.

As a first approximation, we have analyzed the velocity field assuming circular motions dominate. The methods of Warner, Wright, \& Baldwin (1973), van Moorsel \& Wells (1985), and Begeman (1989) were used (see Buta \& Purcell 1998). The van Moorsel and Wells technique and the Begeman technique are coded as GAL and ROCUR, respectively, in the AIPS software package. Use of the three methods allows us to gauge some of the uncertainty in the derived kinematic parameters. The parameters so derived are listed in Table 3 and include the systemic velocity $V_{s}$, the 

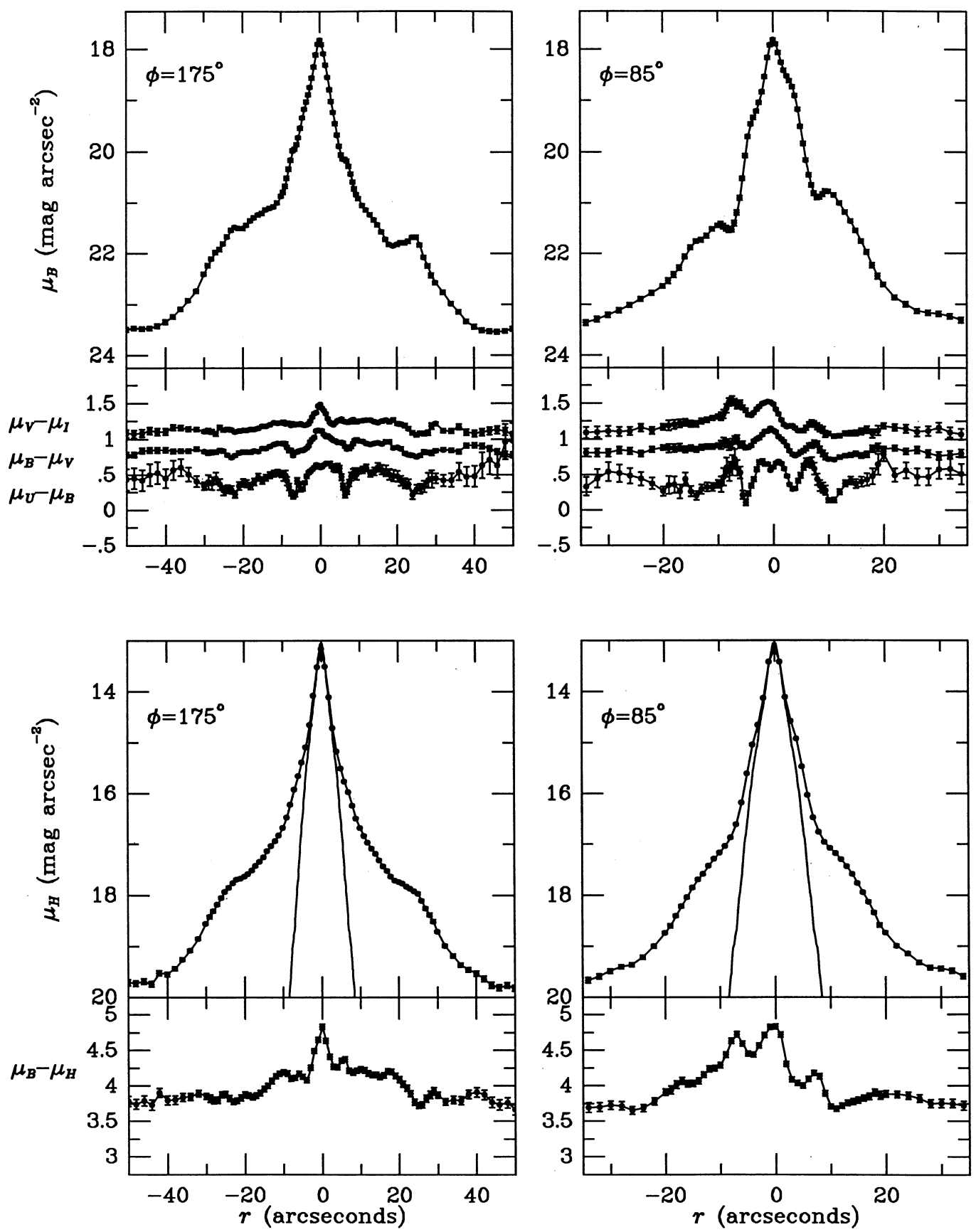

FIG. 5.-Optical and near-infrared luminosity and color index profiles in the inner regions of IC 4214 along the photometric major axis $\left(\phi=175^{\circ}\right)$ and the photometric minor axis $\left(\phi=85^{\circ}\right)$. The solid curves without points in the bottom panels show the $H$-band photometric model of the bulge component, based on the iterative method of Kent (1986). Error bars are based on the gain and read noise of the detector, photon counting statistics, and estimated errors in the determination of the sky level.

TABLE 3

Kinematic Parameters of IC 4214

\begin{tabular}{llll}
\hline \hline \multicolumn{1}{c}{ Parameter } & \multicolumn{1}{c}{ Value $^{\mathrm{a}}$} & \multicolumn{1}{c}{ Value $^{\mathrm{b}}$} & \multicolumn{1}{c}{ Value $^{\mathrm{c}}$} \\
\hline$V_{s}\left(\mathrm{~km} \mathrm{~s}^{-1}\right) \ldots \ldots \ldots$ & 2310 & 2312 & 2310 \\
$\phi_{k}(\mathrm{receding}) \ldots \ldots$ & $169^{\circ}$ & $170^{\circ}$ & $168^{\circ}$ \\
$i_{k} \ldots \ldots \ldots \ldots \ldots \ldots$ & $57^{\circ}$ & $55^{\circ}$ & $58^{\circ}$ \\
$\Delta(\alpha) \ldots \ldots \ldots \ldots \ldots$. & $0^{\prime \prime} 4$ west & $0^{\prime \prime} 3$ west & $0^{\prime \prime}$. \\
$\Delta(\delta) \ldots \ldots \ldots \ldots \ldots$ & $0^{\prime \prime} 3$ south & $0^{\prime \prime} 4$ north & $0^{\prime \prime} .2$ south
\end{tabular}

${ }^{a}$ Based on iterative method of Warner et al. 1973.

b Based on AIPS ROCUR task (Begeman 1989).

c Based on AIPS GAL task and a Brandt model rotation curve (van Moorsel \& Wells 1985). kinematic inclination $i_{k}$, the kinematic line of nodes $\phi_{k}$, and the offsets of the rotation center in arcseconds from the continuum nucleus. The rotation center is found to be very close to the continuum center and within the uncertainties is basically coincident with the observed nucleus. The parameters in Table 3 from ROCUR are based on unweighted averages over 12 radius ranges having roughly the same number of velocity points. The three methods are in good agreement on the derived parameters.

Table 2 summarizes the adopted values of the heliocentric systemic velocity, the inclination, and the line-ofnodes position angle. It is noteworthy that the kinematically derived inclination is $5^{\circ}-10^{\circ}$ larger than the 

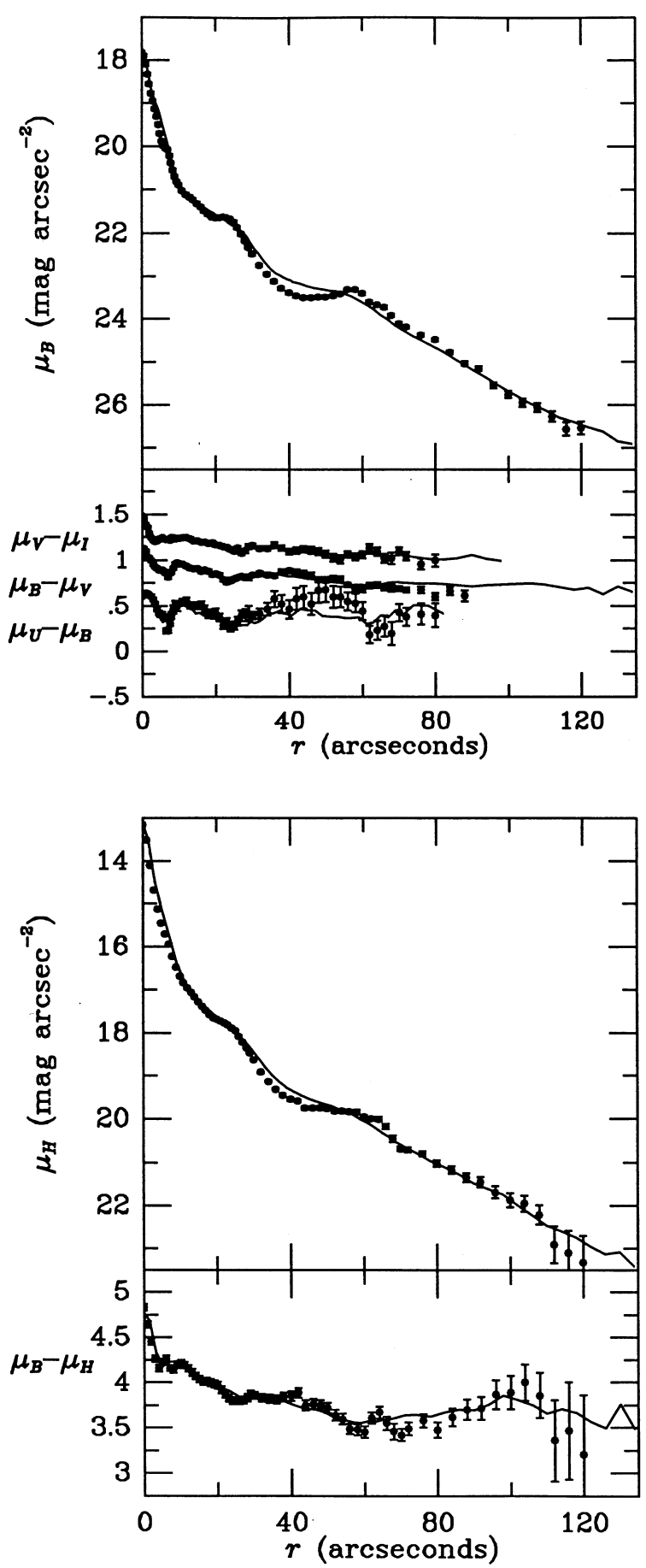

FIG. 6.-Folded major-axis luminosity and color profiles for optical and infrared passbands (filled circles). The error bars on these points are based on the gain and read noise of the detector, photon counting statistics, and estimated errors in the determination of the sky level. The solid curves represent the elliptically averaged profiles based on photometric orientation parameters from Table 1 .

photometric value. The photometric major-axis position angle also disagrees with the kinematic value by $3^{\circ}-6^{\circ}$. We interpret these differences in Paper II.

The unfolded rotation curve based on points within $45^{\circ}$ of the major axis and using the adopted kinematic parameters from Table 2 is shown in Figure 9. This shows that the rotation velocity rises rapidly to a maximum near the radius of the nuclear ring on both sides, after which the rotation velocity decreases. The rotation maximum/minimum on either side suggests the presence of a rapidly rotating nuclear disk of gas. To check whether these regions connect directly to the major axis of the nuclear ring, we have visually mapped the nuclear ring in the $\mathrm{H} \alpha$ emission map. The ring is found to have a diameter of $11^{\prime \prime} .8$, an axis ratio of 0.72 , and a position angle of $171^{\circ}$ (B1950.0). For comparison, the rotation peaks are $17^{\prime \prime} .0$ apart in position angle $168^{\circ}$ (B1950.0). Thus, the nuclear ring is confined entirely within the rotation curve maximum.

The rotation curve shows reasonable symmetry between the two halves of the major axis; therefore, we have folded it about this axis. Figure 10 shows the effect of the uncertainties on our adopted orientation estimates on the shape of the folded rotation curve. The rotation curve based on the kinematic orientation parameters from Table 2 is shown by the filled circles (case 1), while the rotation curve based on the photometric orientation parameters from Table 2 is shown by the crosses (case 2). The solid curve in this plot shows the rotation curve that is derived by using the kinematic line of nodes $\phi_{k}$ in combination with the photometric inclination $i_{p}$ (case 3 ), a combination favored by the analysis of Paper II. Not surprisingly, the shapes of the rotation curves for cases 1 and 2 are rather different. Even though cases 1 and 3 have the same line-of-nodes position angle, varying the inclination changes the shape of the curve beyond a mere scaling because of the incomplete coverage of velocity points over the disk and the presence of noncircular motions. We do not pretend that any of these curves are more correct than the others, but the analysis shows there is much more uncertainty in the rotation curve than the relative scatter of the points would suggest.

\section{DEPROJECTED STRUCTURE OF IC 4214}

In this final section, we examine the approximate deprojected structure of IC 4214, in particular to determine what the deprojected shapes and bar alignments are. This is of interest because statistical studies of apparent axis ratios and relative bar/ring position angles have indicated that inner and outer rings in SB galaxies have an intrinsic axis ratio of $\approx 0.8$ and are aligned perpendicular to each other with the inner ring aligned with the bar (Buta 1995). IC 4214 is not strongly barred, but apart from this it very much resembles a barred, ringed galaxy. Figure 11 shows a montage of deprojected $B$-band images of IC 4214. These were produced using the IRAF routine IMLINTRAN, and for three of the images the range of orientation parameters defined by cases 1-3 in Figure 10 were used for the deprojection. Each image has been rotated to place the bar axis along the horizontal direction. The amount of rotation was determined by first subtracting the bulge model shown in Figure 5 from the $H$-band image, deprojecting the net disk light, adding back the bulge light, and then fitting ellipses to the deprojected isophotes, using isophotes having $35^{\prime \prime}<a<40^{\prime \prime}$ to define the bar (which is mostly an oval). Cases 1-3 are in the top left and bottom panels of Figure 11. Although all three cases favor oval intrinsic shapes for the inner and outer pseudorings, the two rings are not aligned perpendicular to each other for any of the combinations.

To determine which parameters may be consistent with perpendicular alignment, we visually mapped the inner and 

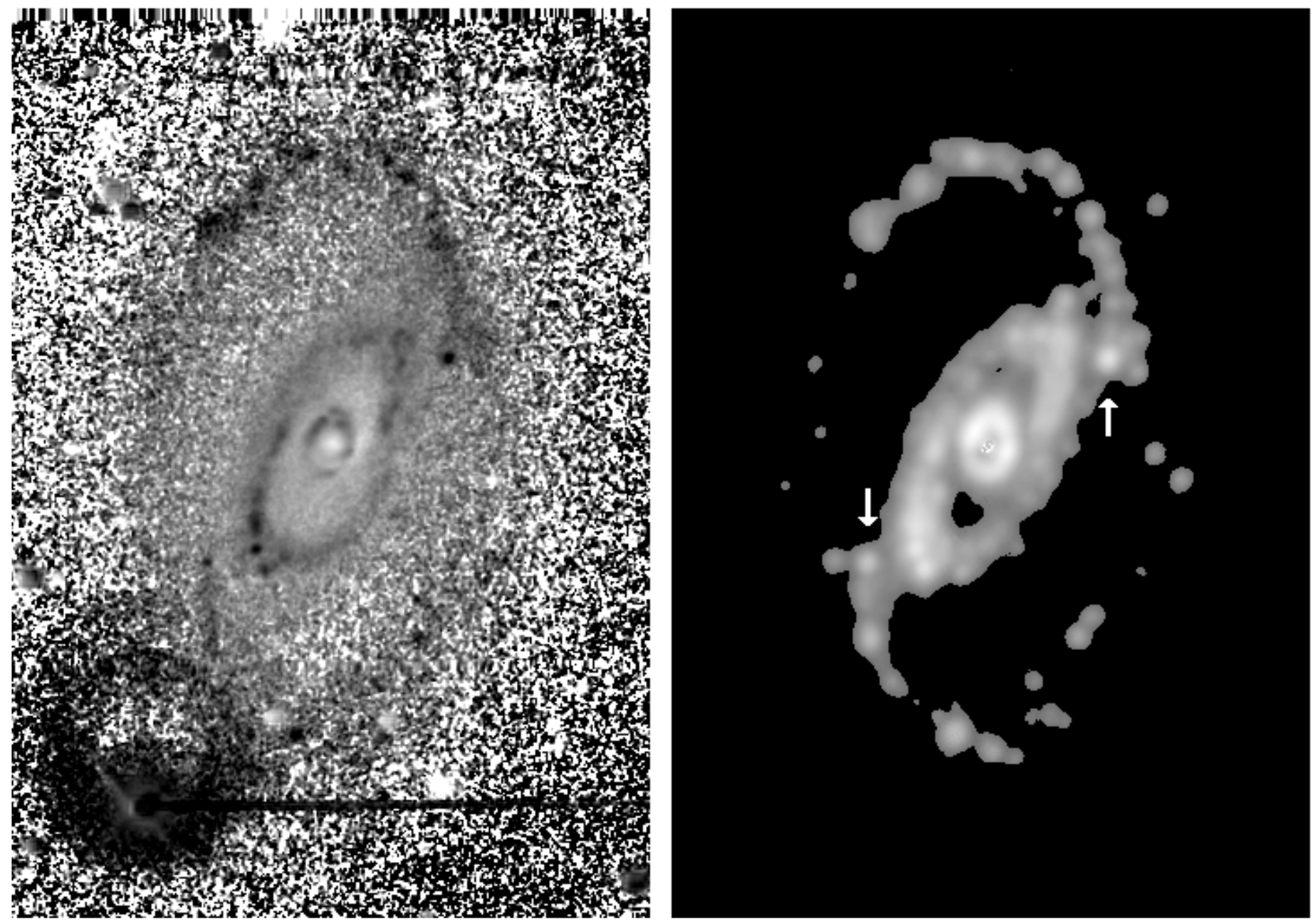

FIG. 7.-Left: $U-I$ color index map showing large-scale star formation distribution. Range shown is from $U-I=1.00$ (darkest pixels) to 3.50 (lightest pixels). Right: $\mathrm{H} \alpha$ emission map from Fabry-Perot interferometry. Arrows point to "dimple" $\mathrm{H}$ in regions discussed in the text. The field shown in each frame covers $3^{\prime} \cdot 3 \times 2 \cdot 3$. North is at the top, and east is to the left.

outer pseudorings and deprojected the mappings using the kinematic line of nodes as a constraint. We found that an inclination of $52^{\circ}$ deprojects the two rings into a perpendicular alignment (see top right panel of Fig. 11). This is well within the uncertainties of the measured inclination, and we suggest that $52^{\circ}$ may be the correct choice. We will refer to the combination $i=52^{\circ}, \phi_{n}(\mathrm{~B} 1950.0)=169^{\circ}$ as the case 4 orientation parameters. Table 4 summarizes the results of ellipse fits to visual mappings of the deprojected inner and outer pseudorings for this case. The deprojected axis ratios are 0.77 for the inner pseudoring and 0.83 for the outer pseudoring, values quite consistent with the intrinsic shapes deduced for SB rings (Buta 1995).

TABLE 4

Deprojected Rings for Case 4 Orientation Parameters

\begin{tabular}{llccc}
\hline \hline \multicolumn{1}{c}{ Feature } & Filter & $\begin{array}{c}\theta_{B, \text { ring }} \\
(\text { deg })\end{array}$ & $\begin{array}{c}a \\
(\operatorname{arcsec})\end{array}$ & \multicolumn{1}{c}{$q$} \\
\hline Nuclear ring $\ldots \ldots \ldots \ldots$. & $\mathrm{H} \alpha$ & 50.1 & 7.0 & 0.84 \\
Nuclear ring .......... & Net $H$ & 48.6 & 7.1 & 0.86 \\
Nuclear ring .......... & $I-H$ & 50.4 & 7.3 & 0.84 \\
Inner pseudoring ....... & $B$ & 1.9 & 28.4 & 0.77 \\
Outer pseudoring ....... & $B$ & 89.9 & 63.8 & 0.83 \\
\hline
\end{tabular}

We illustrate in Figure 12 two other deprojected images of IC 4214 based on the case 4 orientation parameters. The left panel of the figure shows the deprojected $\mathrm{H} \alpha$ map of IC 4214, while the right panel shows the deprojected $H$-band image after subtraction of the bulge model in Figure 5. The $\mathrm{H} \alpha$ map shows that the two "dimple" $\mathrm{H}$ II regions in the outer ring do not perfectly align with the bar axis. Instead, they are roughly symmetrically placed along a line tipped $10^{\circ} \pm 5^{\circ}$ from the bar axis. The image also shows how H II regions in the inner ring roughly bunch up near its intrinsic major axis, similar to what was found for other ringed galaxies by Crocker et al. (1996). H II regions in the outer pseudoring concentrate mainly in the two quadrants that trail the bar axis; that this might be expected for an outer Lindblad resonance feature is shown by the test particle simulations of Byrd et al. (1994). The nuclear ring deprojects into an oval whose major axis is completely misaligned with the bar axis. From a visual mapping, we find an intrinsic axis ratio of 0.84 and an angle of $50^{\circ}$ relative to the bar for this feature (Table 4). The bulge-subtracted $H$-band image is interesting in that the subtraction clearly reveals the nuclear ring as a nearly continuous feature. From a visual mapping, the shape, size, and orientation of this feature are very similar to those for the $\mathrm{H} \alpha$ nuclear ring (Table 4). It is also noteworthy that the bulge-subtracted $H$-band image reveals 


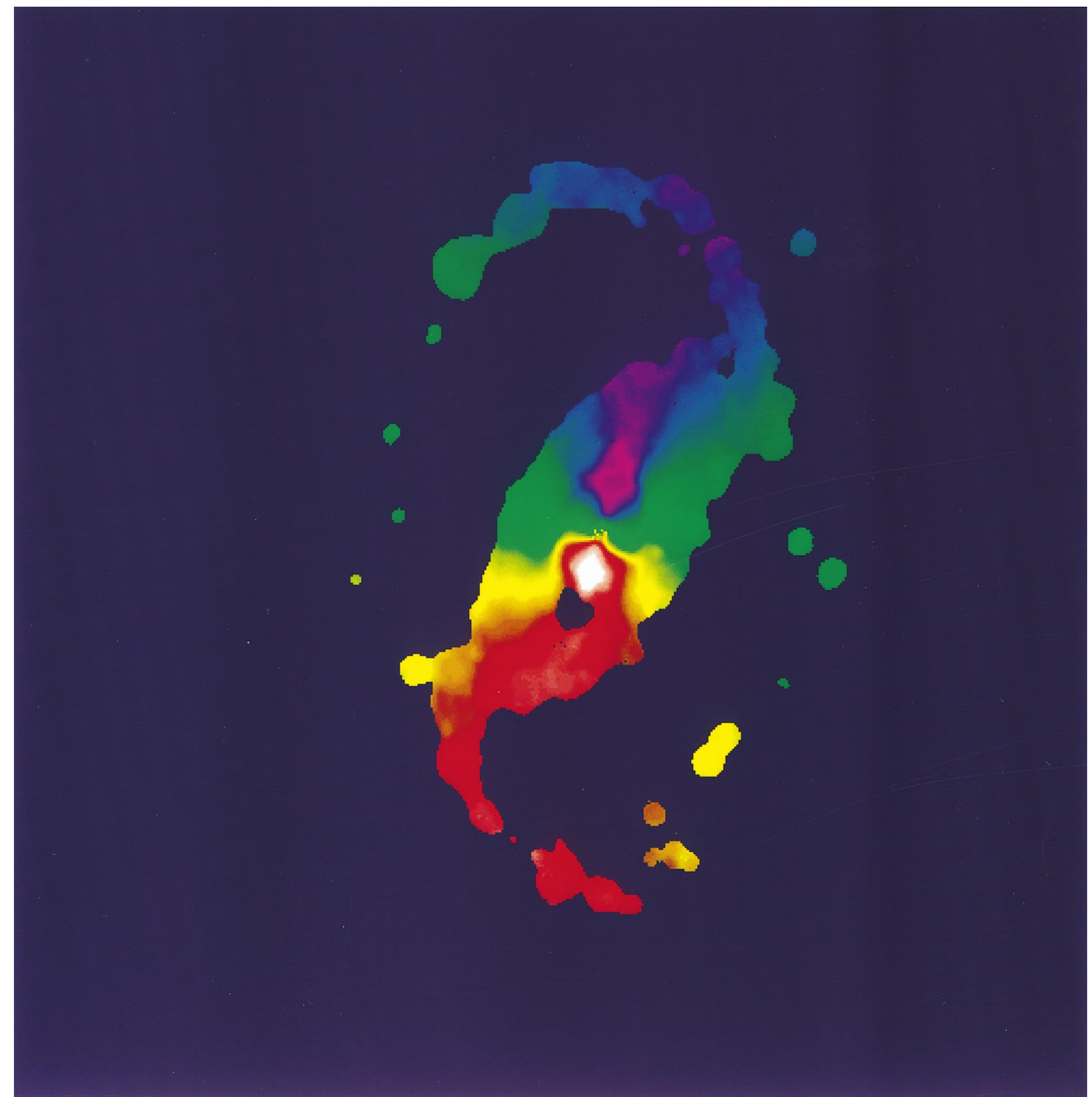

Fig. 8.-Velocity field of IC 4214 from $\mathrm{H} \alpha$ Fabry-Perot interferometry. The color coding is such that white corresponds to a radial velocity of $2530 \mathrm{~km}$ $\mathrm{s}^{-1}$ and purple corresponds to $2090 \mathrm{~km} \mathrm{~s}^{-1}$. The field shown covers $3^{\prime} \cdot 3 \times 3^{\prime} \cdot 4$. North is at the top, and east is to the left.

no evidence for a secondary bar. Such bars are often found inside nuclear rings (see Buta \& Crocker 1993), and since the bulge model is spherical, we would expect to see such a bar if it is present in the residual image.

Finally, we have also deprojected our $I-H$ color index map (see Fig. 13) using case 4 parameters, in order to examine the shape of the red nuclear ring seen in that image as well as to see where the prominent bar dust lanes turn around relative to the bar and inner pseudoring. Table 4 summarizes the results of an ellipse fit to a visual mapping of the red nuclear ring on this deprojected image. It appears that this feature has the same size, shape, and orientation relative to the bar as the nuclear $\mathrm{H} \alpha$ ring. The bar dust lanes turn around at slightly different radii on opposite sides of the center. On the southeast side (Fig. 13. left), the turn around occurs at a radius of 25.7 , while on the northwest side (Fig. 13, right) it occurs at a radius of 22".9. Both values are inside the inner pseudoring whose deprojected majoraxis radius is 28".4. On Figure 13, the southwest dust lane can be traced for $360^{\circ}$ to a maximum radius of $29^{\prime \prime}$. The northwest dust lane may also wind a similar angle; in Figure 13 (left) it can be seen weakly at a radius of 32 "' 8 . The 


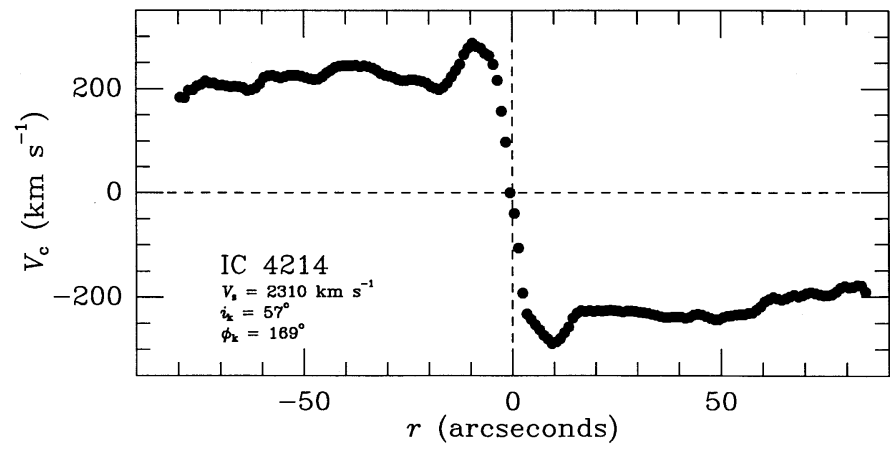

FIG. 9.- Unfolded rotation curve of IC 4214 based on fixed orientation parameters. The curve is shown for an inclination of $57^{\circ}$ and line-of-nodes position angle of $169^{\circ}$ and is based only on points within $45^{\circ}$ of the major axis. Negative velocities are to the north, and positive velocities are to the south.
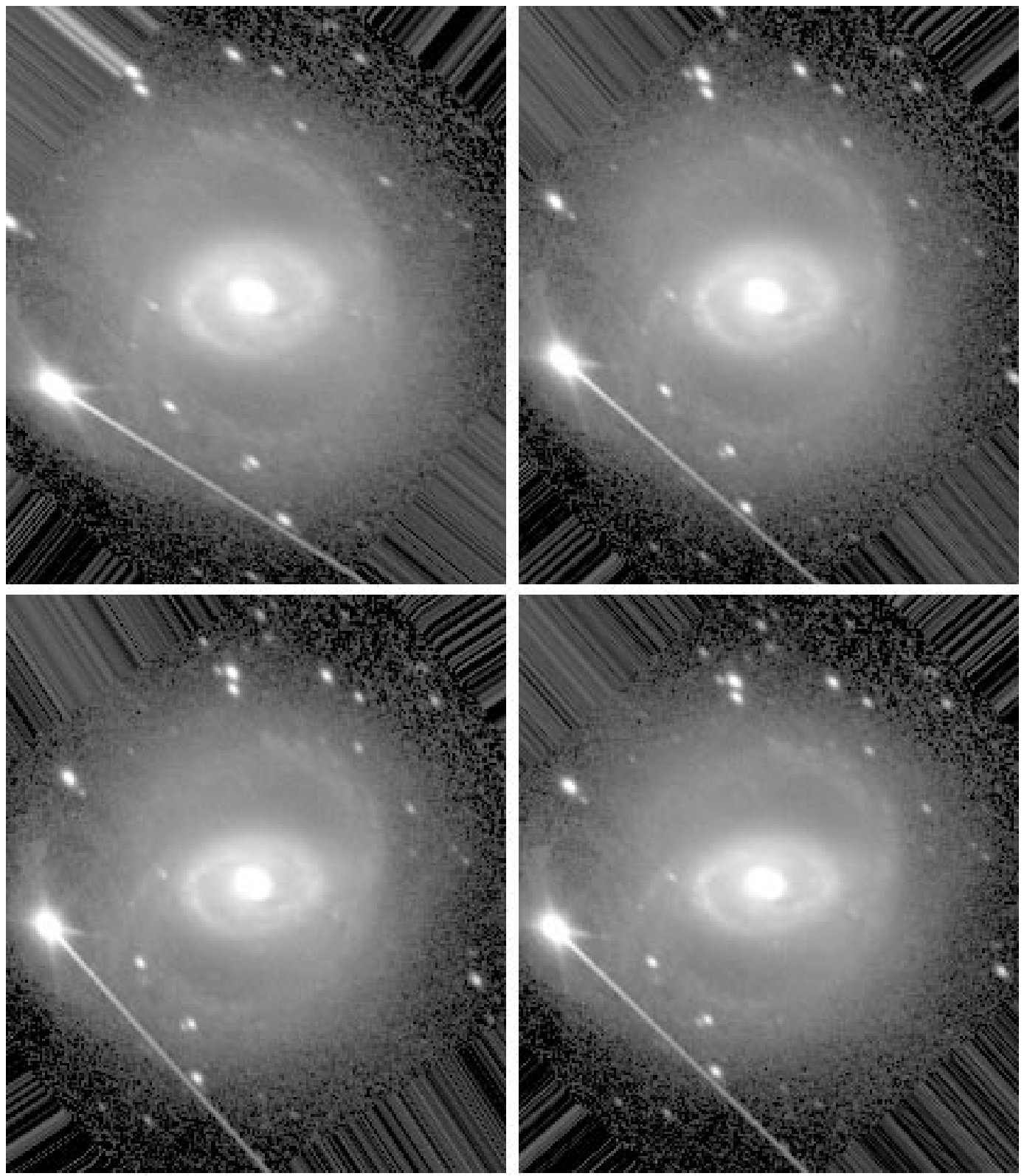

Fig. 11.-Deprojected images for case 1 (top left), case 2 (bottom left), case 3 (bottom right), and case 4 (top right) orientation parameters (see text). All images are in blue light, and all have been rotated to place the bar axis along the horizontal direction. Each frame covers a field of 4.3 $\times 3.7$.
FIG. 10.-Folded rotation curves for the four combinations of orientation parameters, inclination $i$, and line-of-nodes position angle $\phi_{n}$, at bottom right (see text). 

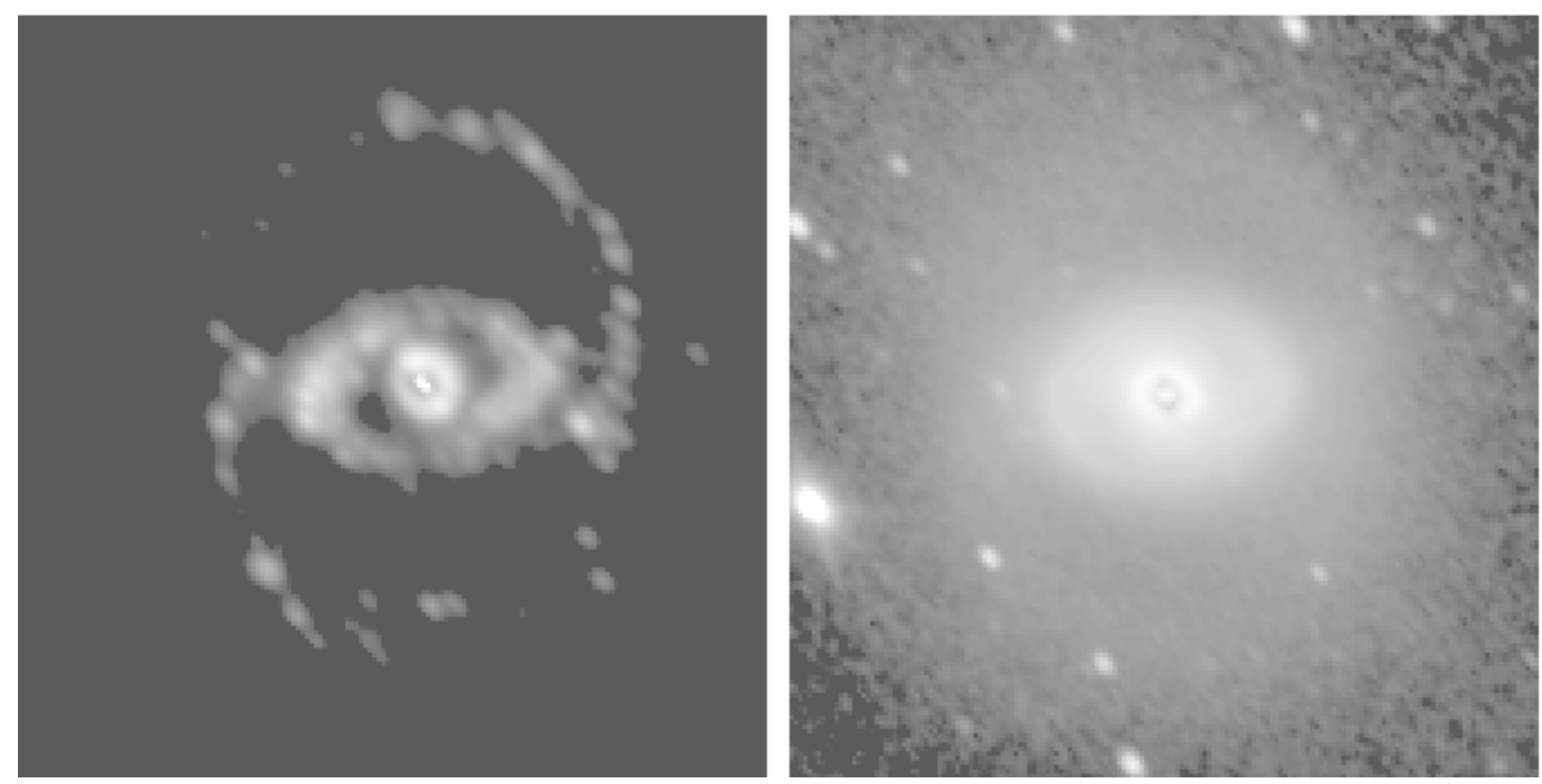

FIG. 12.-Left: Deprojected H $\alpha$ image of IC 4214, using case 4 orientation parameters. Right: Deprojected, bulge-subtracted $H$-band image of IC 4214 for case 4 orientation parameters. In both frames, the image has been rotated to place the bar axis along the horizontal direction. Each frame is 3.2 square.

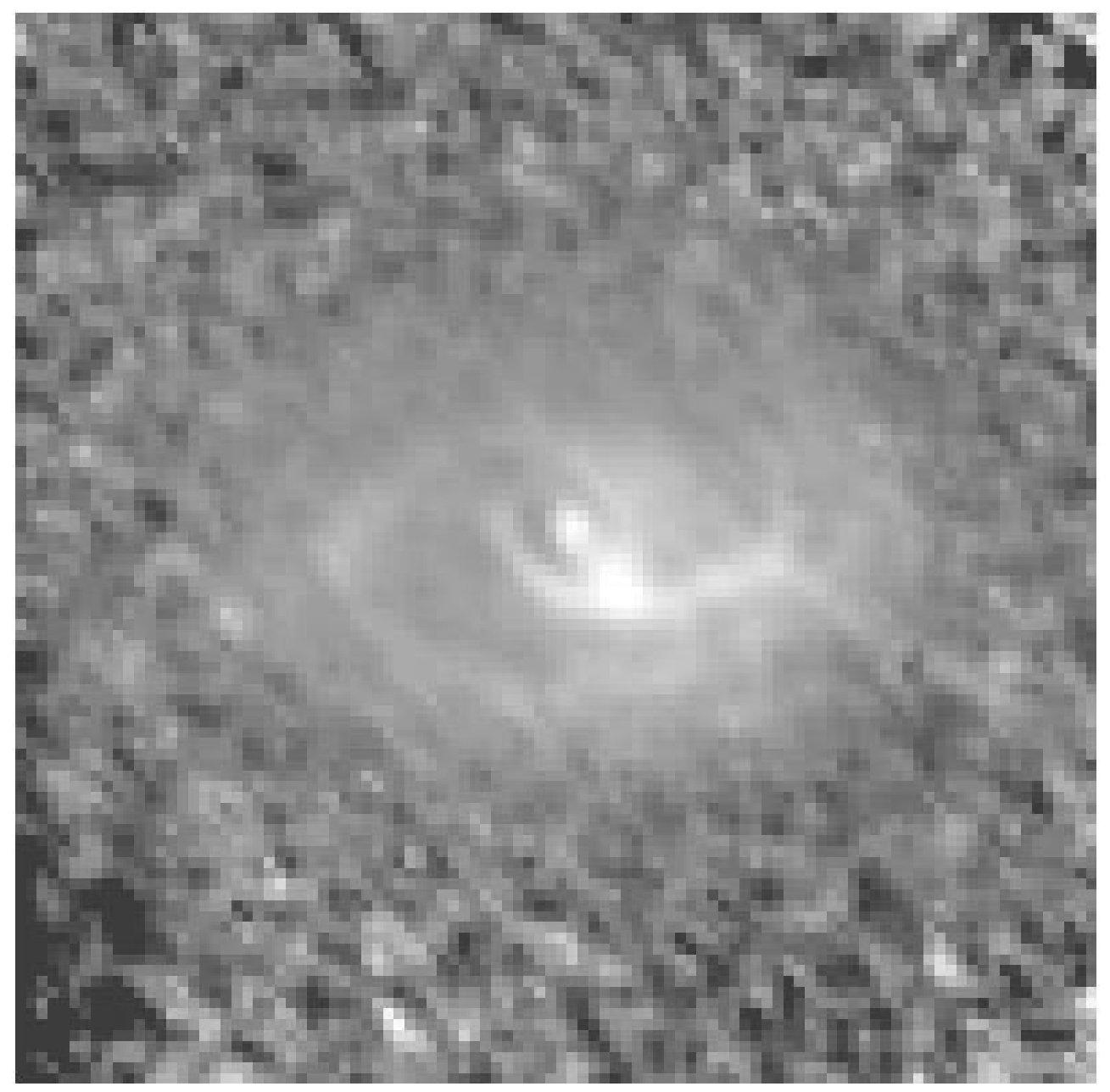

FIG. 13.-Deprojected $I-H$ color index map of IC 4214, using case 4 parameters, to show the pattern of dust lanes in the bar region and the shape of the red nuclear ring. The field shown covers $79^{\prime \prime} \times 79^{\prime \prime}$. 
morphology very much resembles the density response for a homogeneous bar model of low axial ratio $a / b$ shown by Athanassoula (1992, Fig. 7).

\section{SUMMARY}

Our main findings from this paper are summarized as follows:

1. We have shown that IC 4214 is an excellent example of a multiringed early-type, weakly barred galaxy. In blue light, the three rings of IC 4214 are pseudorings made of clear spiral structure. In $\mathrm{H} \alpha$ emission, however, the nuclear ring appears to be a true ring.

2. The bar in IC 4214 is weak, such that it is not easily seen in blue light. Even in the $H$ band, the bar is mostly an oval and in fact is partly defined by a stellar spiral pattern. Although the bar appears weak, an $I-H$ color index map reveals clear dust lanes characteristic of more obviously barred galaxies. These dust lanes curve into a nuclear dust ring and also curve around the ends of the bar.

3 . The three rings of IC 4214 are defined mostly by starforming regions, as is typical of ring phenomena in general. Each ring is a distinct enhancement of blue colors, and each includes $\mathrm{H}$ II regions. Diffuse $\mathrm{H} \alpha$ emission is found within the entire inner oval zone. Prominent $\mathrm{H}$ II regions are found to lie near "dimple" points in the outer pseudoring, where the spiral structure connected with that ring appears to begin.

4. All three rings of IC 4214 are still detectable in the $H$ band, where they are seen as smooth, less spiral-like features. At least for the inner and outer rings, there is an old ring underlying the star-forming pseudoring. The nuclear ring is not easily seen as a ring in the direct $H$-band image, but in a bulge-subtracted image the feature is clearly seen as an oval having a shape and orientation similar to that of the $\mathrm{H} \alpha$ nuclear ring.

5. The velocity field of IC 4214 from $\mathrm{H} \alpha$ Fabry-Perot interferometry is well sampled in the inner regions. The rotation curve shows a rapid rise to a maximum velocity just outside the ridge line of the $\mathrm{H} \alpha$ nuclear ring and then declines to a more constant level, which appears to decline further in the outer regions. The inclination derived from the velocity field differs from the photometrically determined value by possibly as much as $10^{\circ}$, a rather large difference that we attribute in part to noncircular motions in Paper II.

6. In SB galaxies, statistics have indicated that inner and outer rings are in general elongated and aligned perpendicular to each other with the inner ring aligned along the bar axis. When the photometrically derived orientation parameters are used to deproject the blue light image of IC 4214 , the inner and outer rings deproject into ovals that are significantly misaligned from being perpendicular to each other. Misalignment also is found when the kinematically derived orientation parameters are used, and when the kinematic line of nodes and photometric inclination are used in combination (as in Buta \& Purcell 1998 for NGC 3081) instead. We find that in order for the inner and outer pseudorings to deproject into a nearly perpendicular alignment, the kinematic line of nodes in conjunction with an inclination of $52^{\circ}$ works well. This latter value is well within the uncertainties of the inclination determination. For these orientation parameters, the intrinsic shapes, relative sizes, and orientations of the three rings relative to the bar are very similar to what is normally found for SB galaxies.

7. The shapes of dust lanes in the bar region closely resemble those predicted for a homogeneous bar model of low axial ratio $a / b$ (i.e., a " fat" bar).

We thank Ted Williams for assistance with the RFP observations and for providing the essential reduction programs. We also thank an anonymous referee for several helpful suggestions, which improved the paper. R. B., G. B. P., and D. A. C. acknowledge support of NSF grants AST 90-14137 and AST 96-17154 and NSF EPSCoR grant RII 8996152. M. L. C. acknowledges support of NSF Research Experiences for Undergraduates (REU) grant 9424226 .

\section{REFERENCES}

Aaronson, M., Huchra, J., \& Mould, J. 1980, ApJ, 237, 655

Aaronson, M., Huchra, J., Schechter, P. L., \& Tully, R. B. 1982, ApJ, 258, 64

Athanassoula, E. 1992, MNRAS, 259, 345

Begeman, K. G. 1989, A\&A, 223, 47

Bottinelli, L., Gouguenheim, L., Paturel, G., \& de Vaucouleurs, G. 1983, A\&A, 118,4

Buta, R. 1987, ApJS, 64, 1

. 1995, ApJS, 96, 39

Buta, R., Alpert, A. J., Lewis, M., Crocker, D. A., \& Purcell, G. B. 1998, AJ, 116,1142

Buta, R., \& Combes, F. 1996, Fundam. Cosmic Phys., 17, 95

Buta, R., \& Crocker D. A. 1991, AJ, 102, 1715 1992, AJ, 103, 1804 1993, AJ, 105, 1344

Buta, R., \& Purcell, G. B. 1998, AJ, 115, 484

Byrd, G., Rautiainen, P., Salo, H., Buta, R., \& Crocker, D. A. 1994, AJ, 108, 476

Corwin, H. G., de Vaucouleurs, A., \& de Vaucouleurs, G. 1985, Southern Galaxy Catalogue (Austin: Univ. Texas Dept. Astron.)

Crocker, D. A., Baugus, P. D., \& Buta, R. 1996, ApJS, 105, 353

de Vaucouleurs, G. 1963, ApJS, 8, 31

de Vaucouleurs, G., de Vaucouleurs, A., Corwin, H., Buta, R., Paturel, G., \& Fouqué, P. 1991, Third Reference Catalogue of Bright Galaxies (New York: Springer)
Elias, J. H., Frogel, J. A., Matthews, K., \& Neugebauer, G. 1982, AJ, 87, 1029

Fouqué, P., Bottinelli, L., Gouguenheim, L., \& Paturel, G. 1990, ApJ, 349,1

Graham, J. A. 1982, PASP, 94, 244

Hubble, E. 1926, ApJ, 64, 321

Jedrzejewski, R. 1987, MNRAS, 226, 747

Kent, S. M. 1986, AJ, 91, 1301

Landolt, A. U. 1992, AJ, 104, 340

Lauberts, A. 1982, The ESO-Uppsala Survey of the ESO (B) Atlas (Munich: ESO)

Martin, P., \& Roy, J.-R. 1994, ApJ, 424, 599

Martinet, L. 1994, Fundam. Cosmic Phys., 15, 341

Salo, H., Rautiainen, P., Buta, R., Purcell, G. B., Cobb, M. L., \& Crocker, D. A. 1999, AJ, 117, 792 (Paper II)

Saraiva, M. F. 1997, AJ, 113, 1607

Schommer, R. A., Bothun, G. D., Williams, T. B., \& Mould, J. R. 1993, AJ, 105,97

Schwarz, M. P. 1981, ApJ, 247, 77

Sellwood, J. A., \& Wilkinson, A. 1993, Rep. Prog. Phys., 56, 173

Shlosman, I., Frank, J., \& Begelman, M. C. 1989, Nature, 338, 45

Simkin, S., Su, H. J., \& Schwarz, M. P. 1980, ApJ, 237, 404

van Moorsel, G. A., \& Wells, D. C. 1985, AJ, 90, 1038

Warner, P. J., Wright, M. C. H., \& Baldwin, J. E. 1973, MNRAS, 163, 163 